\title{
On the dynamic buckling of very slender structures to self-weight
}

\author{
Reyolando Brasil ${ }^{1, *}$, Leandro Orbolato ${ }^{2}$, Eduardo Pádua $^{1}$ \\ ${ }^{1}$ Federal University of ABC, São Bernardo do Campo, SP, Brazil \\ ${ }^{2}$ UNOESTE, Presidente Prudente, SP, Brazil
}

\begin{abstract}
We present a mathematical and experimental study of the dynamic buckling of very slender structures due to their self-weight. Modern materials and powerful new analysis methods are leading to the design of very slender tall structures that may be prone to instability issues. Elastic stability of such structures is a problem inside the scope of the NonLinear Dynamics Analysis Methods. An indicator of instability is when the structure's free vibration frequency approaches null value. Two main factors affect these frequency results. First the stiffness, composed of elastic stiffness, always positive and non-zero, that diminishes rapidly with height, and the geometric stiffness, negative for compressive forces, whose absolute value grows as the structure gets taller and heavier. Second, the mass, that also grows with the height of the structures. To access this behaviour, we first present a simple one-degree-of-freedom mathematical model derived with Rayleigh's Method, adopting a cubic polynomial as shape function. Next, comparisons are made with results of an experimental set up composed of a variable length cantilever vertical aluminium bar. These models reasonably agree with analytical close solutions available in the literature.
\end{abstract}

\section{Introduction}

In this paper, a mathematical and experimental study of the dynamic buckling of very slender structures due to their self-weight, a classical problem, is revisited. It is an important issue in several applications such as Eolic energy generators and cell phone equipment support towers, industrial chimneys etc.

Modern materials and powerful computational analysis methods, usually based on the Finite Element Method [1], are leading to the design of very slender tall structures that are likely to be prone to instability issues.

Elastic stability of such structures is a problem inside the scope of the Non-Linear Dynamics Analysis Methods. The main issue is the variation of the geometric stiffness.

An indicator of instability is when the structure's free vibration frequency approaches null value. Two main factors affect these frequency results. First the stiffness, composed of elastic stiffness, always positive and non-zero, that diminishes rapidly with height, and the

\footnotetext{
*Corresponding author: reyolando.brasil@ufabc.edu.br
} 
geometric stiffness, negative for compressive forces, whose absolute value grows as the structure gets taller and heavier. Second, the mass, always positive, that also grows with the height of the structures.

In this paper, to access this behaviour, we first present a simple one-degree-of-freedom mathematical model derived with Rayleigh's Method [2], adopting a cubic polynomial as shape function. Next, comparisons are made with results of an experimental set up composed of a variable length cantilever vertical aluminium bar. These models reasonably agree with analytical close solutions available in the literature, e.g., [3] and [4].

\section{Rayleigh's method}

\subsection{Formulation}

John William Strutt, $3^{\text {rd }}$ Baron of Rayleigh, presented, in "The Theory of Sound" [2], a simplified method to access the dynamic properties of structures. In the case of vertical beams, the transverse horizontal vibration displacement of its $x$ axis, as shown in Fig. 2, is a function of both the spatial $x$ coordinate and time, and may be written as

$$
u=u(x, t)=\phi(x) q(t)
$$

where $\phi(t)$ is a dimensionless shape function, that must satisfy the geometric boundary conditions and assume unitary value where the $q(t)$ generalize coordinate is chosen. In our model, Fig. 1, that is the horizontal displacement the top section of the beam, where a point vertical load $P$ may be applied, usually self-weigh of a point mass $M$.

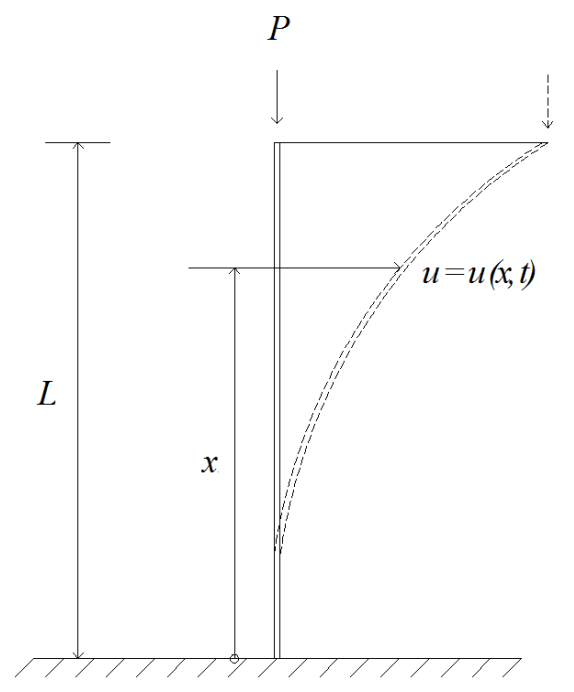

Fig. 1. Mathematical model.

For model of Fig. 1, a prismatic, cantilever, homogeneous beam, with modulus of elasticity $E$, transverse section moment of inertia $I$, mass per unit length $\bar{m}$, self-weight per unit length $q=\bar{m} g$, an adequate such function is a simple cubic polynomial: 


$$
\phi(x)=\frac{3 x^{2}}{2 L^{2}}-\frac{x^{3}}{2 L^{3}}
$$

that displays zero displacement and tangent of its $x$ longitudinal axis at the base and null second derivative at the top, related to zero bending moment there.

The resulting equivalent elastic stiffness is

$$
k_{0}^{*}=E I \int_{0}^{L}\left(\phi^{\prime \prime}\right)^{2} d x=\frac{3 E I}{L^{3}}
$$

the equivalent geometric stiffness, due to the point vertical load $P$ (negative if compressive), is

$$
k_{g}^{*}=P \int_{0}^{L}\left(\phi^{\prime}\right)^{2} d x=\frac{6 P}{5 L}
$$

the equivalent geometric stiffness, due to self-weight, negative, is

$$
\left.k_{g}^{* *}=-q \int_{0}^{L}(L-x)\right)\left(\phi^{\prime}\right)^{2} d x=-\frac{3}{8} q
$$

and the equivalent lumped mass at the top of the beam is

$$
m^{*}=\bar{m} \int_{0}^{L}\left(\phi^{\prime \prime}\right)^{2} d x=\frac{33 \bar{m} L}{140}
$$

The first natural frequency, in radians per second, of this mathematical model, is given by

$$
\omega_{1}=\sqrt{\frac{k}{m^{*}}}
$$

where the total stiffness $k$ is the sum of Eqs. (3), (4) and (5),

$$
k=k_{0}^{*}+k_{g}^{*}+k_{g}^{* *}
$$

\subsection{Numerical results}

Numerical parameters adopted for our simulations are: rectangular transverse section $25.4 \times 3.175 \mathrm{~mm}$, aluminium elasticity modulus $\mathrm{E}=70 \mathrm{GPa}$ and mass per unity volume $2,700 \mathrm{~kg} / \mathrm{m}^{3}$, gravity $10 \mathrm{~m} / \mathrm{s}^{2}$.

In Table 1 we present variation of the first natural frequency with the $L$ free length of the cantilever beam if no point vertical force $P$ at the top is considered.

One will note that zero frequency, corresponding to buckling due to self-weight, occur for $L$ $=2.5924 \mathrm{~m}$, only $0.7 \%$ larger than the value $\mathrm{L}=2.5747 \mathrm{~m}$ given by the analytical result [3] . 
Table 1. Numerical simulations of Rayleigh's mathematical model $(P=0)$.

\begin{tabular}{|c|c|}
\hline Free length (m) & Frequency ( $\mathbf{r a d} / \mathbf{s})$ \\
\hline 2.0 & 3.061 \\
\hline 2.1 & 2.584 \\
\hline 2.2 & 2.145 \\
\hline 2.3 & 1.729 \\
\hline 2.4 & 1.314 \\
\hline 2.5 & 0.856 \\
\hline 2.5924 & 0 \\
\hline
\end{tabular}

\section{Experimental measurements}

\subsection{The experimental set up}

A very simple experimental set-up was built using a commercial aluminium bar with the same characteristics as those adopted for the mathematical model, that is, rectangular transverse section $25.4 \times 3.175 \mathrm{~mm}$, aluminium elasticity modulus $\mathrm{E}=70 \mathrm{GPa}$ and mass per unit volume $2,700 \mathrm{~kg} / \mathrm{m}^{3}$.

The bar full length was procured to be $3.0 \mathrm{~m}$. Next, the bar was clamped by a bench vise leaving several free length values. For each case, free vibrations were induced by nontrivial initial conditions at the top. Frequencies were measured by a commercial data acquisition system connected to a notebook computer, picked up from a regular electric strain gage glued to the bar. Photos of this set up are displayed in Figs. 2 and 3.

\subsection{Experimental results}

Table 3 presents variation of the measured first natural frequencies with the $L$ free length of the cantilever beam. One will note that results are larger than given by the analytical result in [3] and the mathematical model, due to inevitable experimental imperfections. We also present the percental variation of frequency values for the mathematical model and the experimental measurements. As expected, differences get larger as one approaches the buckling load. 


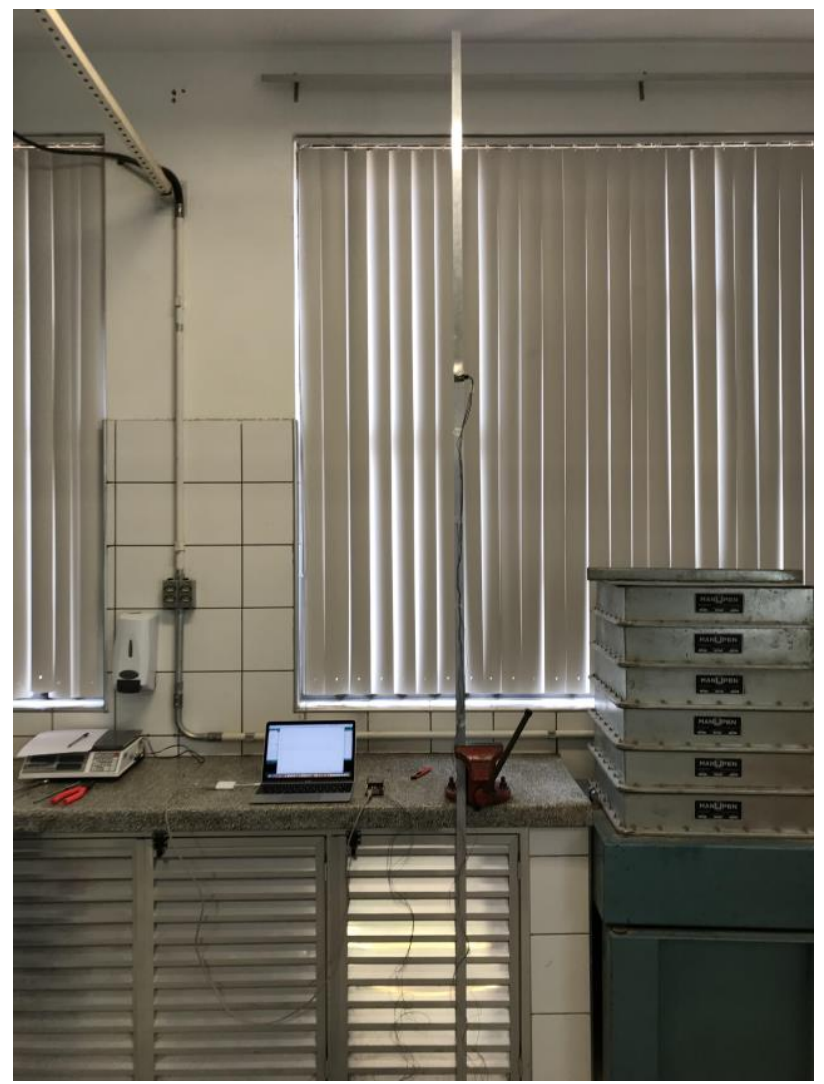

Fig. 2. Experimental set up.

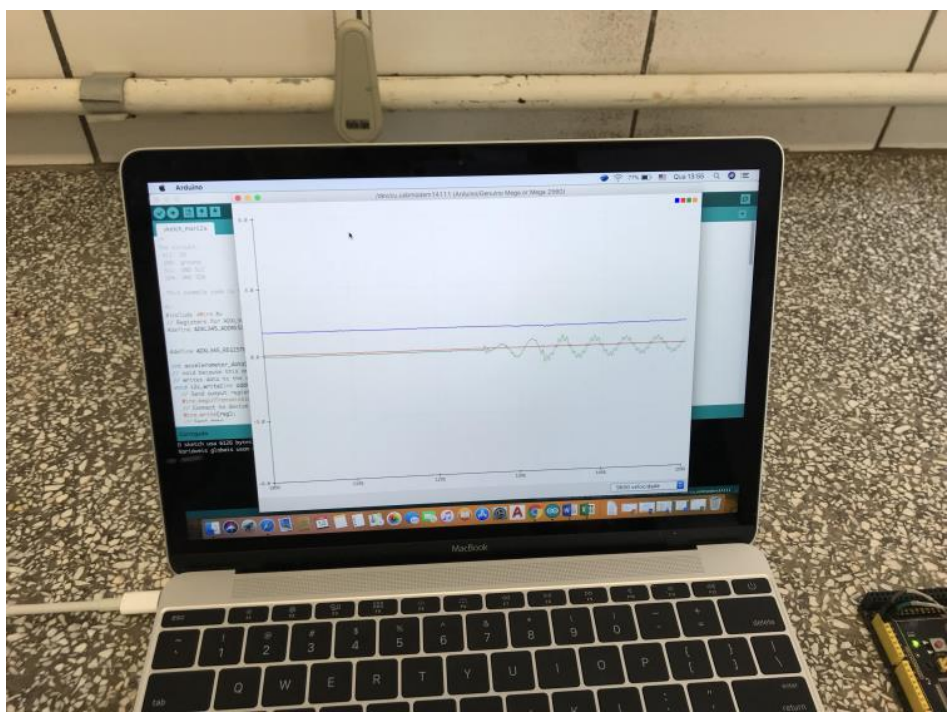

Fig. 3. Data acquisition and treatment. 
Table 3. Experimental results and comparisons.

\begin{tabular}{|c|c|c|c|}
\hline Free length (m) & $\begin{array}{c}\text { Frequency (rad/s) } \\
\text { Experimental }\end{array}$ & $\begin{array}{c}\text { Frequency (rad/s) } \\
\text { Mathematical }\end{array}$ & Percental difference \\
\hline 2.0 & 3.141 & 3.061 & 2.61 \\
\hline 2.1 & 2.673 & 2.584 & 3.44 \\
\hline 2.2 & 2.243 & 2.145 & 4.56 \\
\hline 2.3 & 1.903 & 1.729 & 10.06 \\
\hline 2.4 & 1.514 & 1.314 & 15.22 \\
\hline 2.5 & 1.131 & 0.856 & 32.12 \\
\hline
\end{tabular}

\section{Conclusions}

In this paper, we revisited the classical elastic stability problem related to buckling due to self-weight of a cantilever column, focusing on the variation of its first natural frequency with length, due to the variation of geometric stiffness. Zero frequency indicates lost of stability. Results of a mathematical model derived with Rayleigh's method, using a cubic shape function, were compared to experimental measurements and analytical solutions available in literature. Experimental results are larger than given by the analytical reference value in [3] and the mathematical model, due to inevitable experimental imperfections. As expected, differences get larger as one approaches the buckling load.

The author acknowledges support by FAPESP, and CNPq, both Brazilian research funding agencies.

\section{References}

[1] R.W. Clough, J. Penzien, Dynamics of Structures, $2^{\text {nd }}$ Ed, McGraw-Hill, New York, (1993)

[2] J.W.S. Rayleigh, The theory of sound, Dover, London, (1945).

[3] S.P. Timoshenko, J. M. Gere, Theory of elastic stability, $2^{\text {nd }}$ Ed, McGraw-Hill, New York, (1961)

[4] Z.P. Bazant, L. Cedolin, Stability of Structures: Elastic, Inelastic, Fracture and Damage Theories, World Scientific, Danvers, (2010) 